\title{
The TMT/NFIRAOS LGS wavefront sensing demonstration bench
}

\author{
R.Conan ${ }^{1, a}$, O. Lardière ${ }^{1}$, G. Herriot ${ }^{2}$, C. Bradley ${ }^{1}$, and K. Jackson ${ }^{1}$ \\ 1 Adaptive Optics Laboratory, University of Victoria \\ 2 NRC-Herzberg Institute of Astrophysics, Victoria, Canada
}

\begin{abstract}
LGS wavefront sensing on ELTs is challenging. The tip-tilt indetermination, the cone effect, the star elongation, the sodium profiles fluctuations are the main problems plaguing LGS wavefront sensors. The AO Laboratory at the University of Victoria has build a LGS wavefront sensing test bed scaled for the TMT, reproducing the sodium variations and the whole NFIRAOS wavefront sensing scheme. The details of the bench design are given and the implementation of the wavefront sensing algorithm is presented. This one includes a matched filter, zoom optics control, DM command and truth wavefront sensors management.
\end{abstract}

\section{Introduction}

Both next largest optical telescopes, the TMT and the E-ELT, will equipped their AO systems with Laser Guide Stars (LGS). When seen through a SH-WFS conjugated to the primary mirror of an ELT, the spot elongation away from the LGS launch axis is large enough to resolve the fluctuations in the $\mathrm{Na}$ layer profiles.

Fig. 1 (a) shows the imaging process of a LGS with a Shack-Hartmann WFS. The thickness of the Na layer induces a LGS spanning the whole layer. The lenslets of the SH-WFS on the side of the telescope primary mirror are imaging the LGS sideways producing an elongated spot. The elongated spot intensity is proportional to the $\mathrm{Na}$ density profile in the $\mathrm{Na}$ layer. The Na profile is varying with time hence affecting the centroid of the elongated spot of an non-optical error. The temporal controller of a closed-loop AO system will perceive this error as an optical error attempting to correct for it with the DM adding new optical aberration in the science path.

New centroiding schemes have been derived to overcome the LGS induced aberrations problem. Most of these algorithms have been tested with end-to-end simulation. In this paper, an optical test bed is presented which reproduces the features of a LGS guide star as seen from a SH-WFS at an ELT.

\section{The Bench}

The Adaptive Optics Laboratory of the University of Victoria has built an optical test bench[6] dedicated to LGS wavefront sensing. This bench is tailored to NFIRAOS[5], the TMT[2] AO facility. It has the following features:

- spot elongation of 4" (8 pixels)

- dynamic zoom to focus on mean Na layer altitude

- sodium density variations (spatial \& temporal)

- dithering of the spots for the matched filter

- able to generate some atmospheric turbulence

- combine both LGS and NGS WFSs

Fig. 2 is a picture of the bench layout with the two WFSs, the DM and the two sources. The LGS WFS has $29 \times 29$ lenslets with $15 \times 15$ pixels for each. The NGS WFS has $12 \times 12$ lenslets with $8 \times 8$

\footnotetext{
a e-mail: rconan@uvic.ca
} 
Fig. 1. LGS imaging with a Shack-Hartmann WFS on an ELT (left) and in the Lab (right).

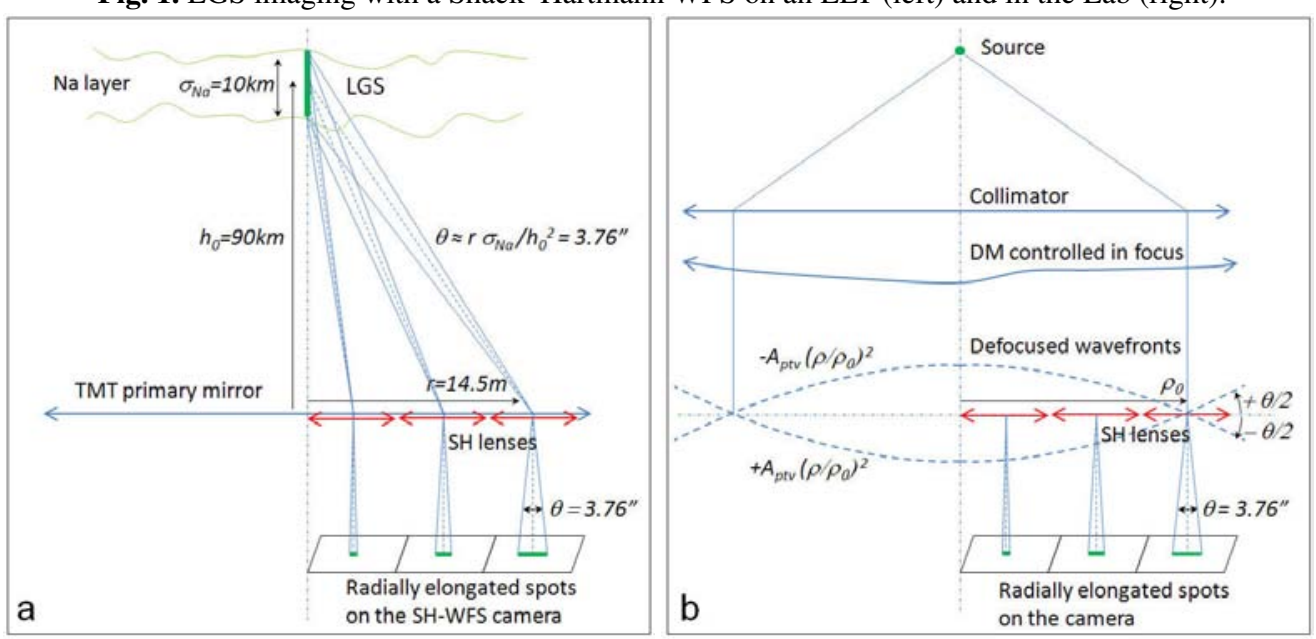

Fig. 2. The UVic AO Lab LGS wavefront sensing test bench.

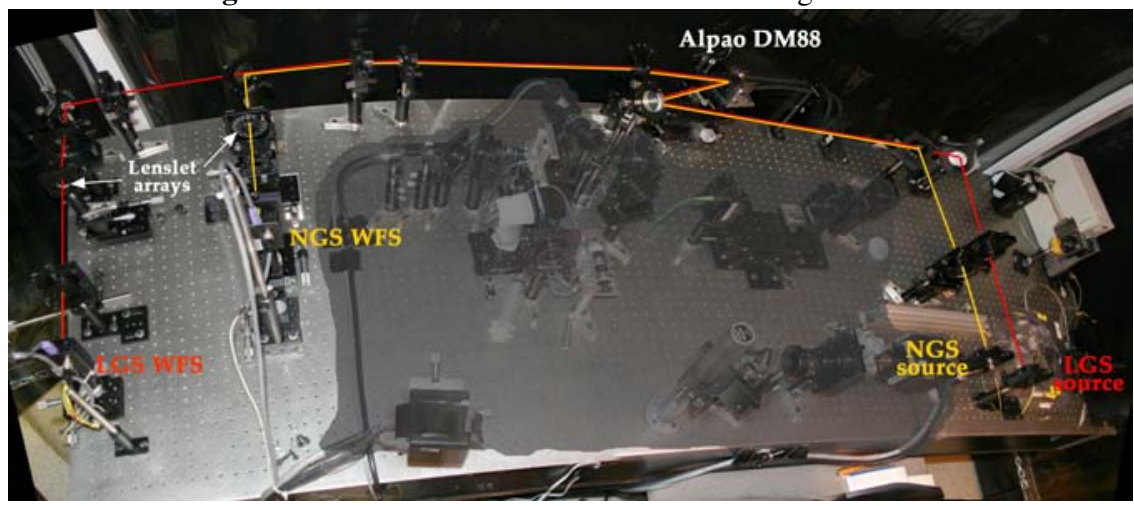

pixels for each. The DM is a magnetic ALPAO DM with 88 actuators in the pupil on a $10 \times 10$ regular grid. The two sources are point like Laser diode at $600 \mathrm{~nm}$ and $700 \mathrm{~nm}$.

Fig. 1(b) describes the process to make elongated spot on the test bench. To create the spot elongation, a focus mode is applied to the DM. The amplitude of the focus alternates between two $\mathrm{min} / \mathrm{max}$ values. Consequently, the point like spots sweep the detector while the electronic shutter remains open during the sweeping. After running an entire range a focus, the camera is read out giving the elongated spots. The elongation size depends on the distance of the lenslet from the optical axis. Fig. 3 shows spot images produced with the bench. On the left, it is an image for a LGS launch on-axis from a 30m telescope and a $20 \mathrm{~km}$ thick $\mathrm{Na}$ layer. On the right, it is an image for a side launch Laser and a $7.5 \mathrm{~km}$ thick Na layer on a $42 \mathrm{~m}$ telescope.

Using Na profiles from UBC Lidar, the performance of different centroiding algorithms has been compared. Fig. 4 shows the rms of ptv Zernike modes for a center-of-gravity (CoG) and a matched filter (MF) $[4,1]$. These errors are obtained by reproducing a time sequence of Na profiles and projecting the slopes deduced from both algorithms onto the Zernike polynomials. It demonstrates how the MF mitigates the LGS aberrations.

Fig. 5 shows the centroiding error as a function of the SNR measured on an non-elongated spot. It compares non-thresholded $\left(\mathrm{CoG}_{0 \%}\right)$ and thresholded $\left(\mathrm{CoG}_{8 \%}\right) \mathrm{CoG}$ algorithms to a standart $\mathrm{MF}$ and a noise-weighted $\mathrm{MF}[3]\left(\mathrm{MF}_{\eta}\right)$. The noise-weighted $\mathrm{MF}$ gives the smallest error especially at low SNR. It means too that for a given error when using $\mathrm{MF}_{\eta}$, one can work at a lower SNR than the CoG allowing to save, for example, costly Laser power. 
Fig. 3. Central(left) and side(right) launch LGS reproduced with the bench.

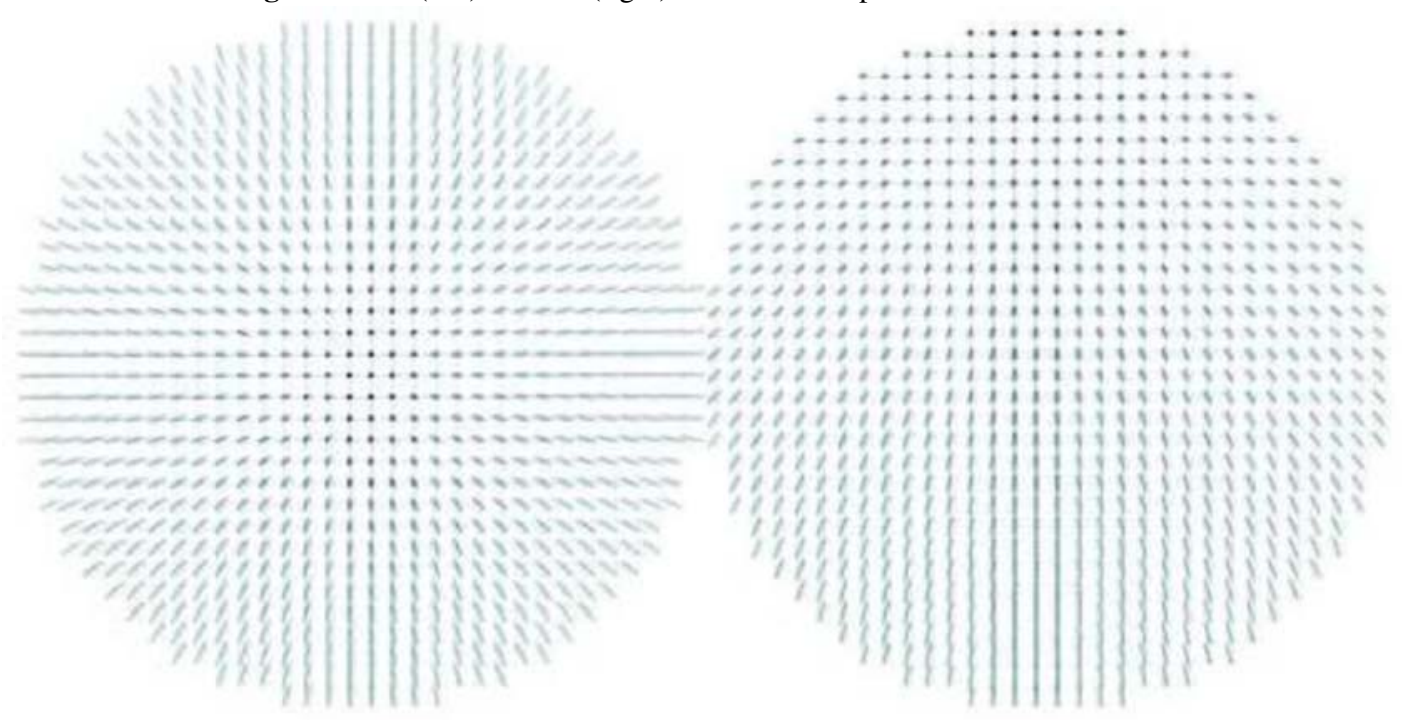

Fig. 4. LGS aberrations

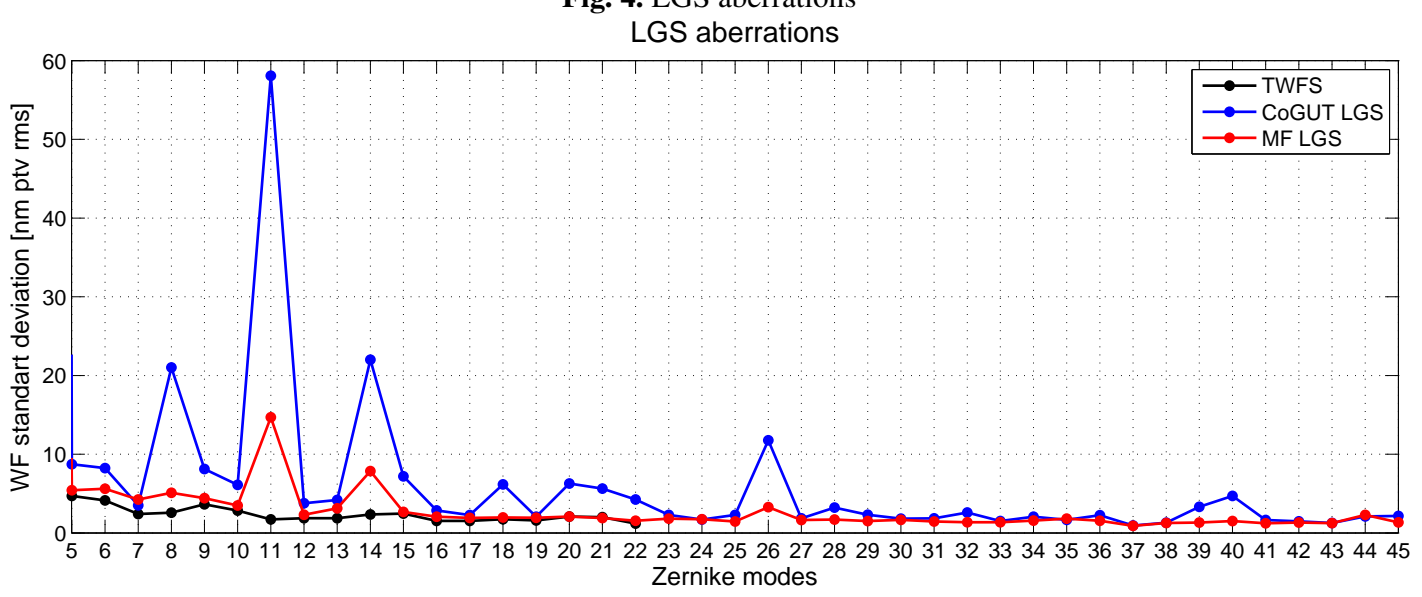

\section{NFIRAOS LGS wavefront sensing control system}

The LGS wavefront sensing bench has been used to implement and to validate the whole NFIRAOS LGS/NGS wavefront sensing control system. Fig. 6 shows the complete control system. Apart the LGS WFS, two NGS WFS are used. The on-instrument tip-tilt-focus WFS (OI TTF WFS) is a fast SH-WFS measuring only tip-tilt and focus modes. The medium order WFS (MOR WFS) is a slow WFS measuring low order modes above focus. A fast steering mirror (FSM) is used to stabilize and to dither the Laser. A zoom optics re-focus the light to infinity. And the DM off-course corrects the phase aberrations.

On the bench, a single $12 \times 12$ NGS WFS is used to emulate both OI TTF WFS and MOR WFS. The NGS WFS is run as fast as the LGS WFS. The slopes are projected onto Zernike modes. The Zernike modes above the focus are time averaged to reproduce the slow MOR WFS measurements. The FSM and DM are both emulated with a single ALPAO DM. The zoom optics is reproduced by numerically shifting the barycenter of the Na profiles. 


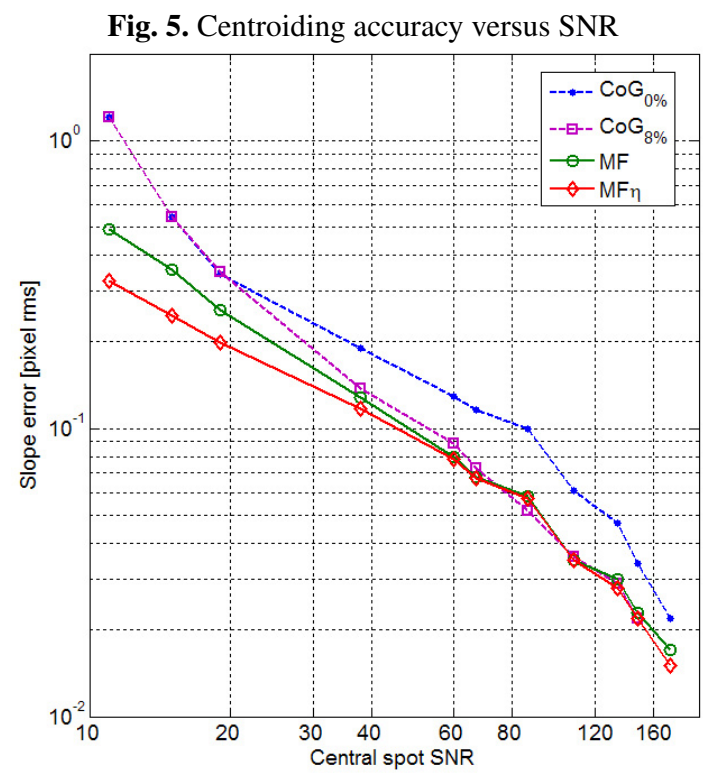

The rejection transfer functions of the system have been derived analytically. Fig. 7 shows the rejection transfer functions of the turbulence and of the LGS aberrations. As the data of the LGS WFS and of the MOR WFS are blended together, the update rate $f_{I O}$ of the MF reference image and gain and the frame rate $f_{M O R}$ of the MOR WFS have to be chosen carefully.

The MOR and LGS WFS data are added together the following way $g_{I}\left(L G S+g_{M O R} M O R\right) . g_{I}$ is the gain of the DM integrator and $g_{M O R}$ is the gain of the MOR WFS. $g_{M O R}$ will affect the settle time and the stability of the closed loop system.

In Fig. 7, it can be seen that when $f_{I O}=f_{M O R}$, the DM/LGS+MOR system behaves like a DM and single WFS system. If the MOR WFS frame rate is too slow with respect to the MF update rate, low frequency aberrations are under-corrected. The mathematical model has been checked against bench results. Fig. 8 shows bench measured rejection transfer functions for the two same cases plotted in Fig. 7. It confirms that when both frequencies are the same, the system acts like a one WFS closed loop AO system. Whereas with a slower MOR WFS, the low frequency correction is poorer.

\section{Conclusion}

The Adaptive Optics Laboratory of the University of Victoria has built a optical test bed for LGS wavefront sensing. The bench reproduces the spot elongation and the Na layer fluctuation. For NFIRAOS, the TMT AO facility, the center-of-gravity and matched filter centroiding algorithms ability to mitigate LGS aberrations have been compared. It has been shown that the MF is able to track the LGS aberrations while correcting for the turbulence. In addition, for a given wavefront error the MF can work at a lower SNR than the CoG.

The whole DM/NGS+LGS closed-loop control system of NFIRAOS has also been implemented on the bench. It has allowed to validate and to improve the different algorithms and control design choices. It has been shown that the best performance are obtained when the update rate of the MF and the frame rate of the MOR WFS are the same. The gains of the control system can be optimized based on the analysis of the transfer functions of the system computed from an analytical model of NFIRAOS control system. The model has been found to be in good agreement with the transfer functions measured with the bench.

A new LGS WFS tailored to the E-ELT specifications is going to be integrated into the bench. It will allow to compare how centroiding algorithms like CoG, MF, quad-cell, weighted CoG and correlation mitigate the LGS aberrations for central and side launch Laser. 
R.Conan et al.: The TMT/NFIRAOS LGS wavefront sensing demonstration bench

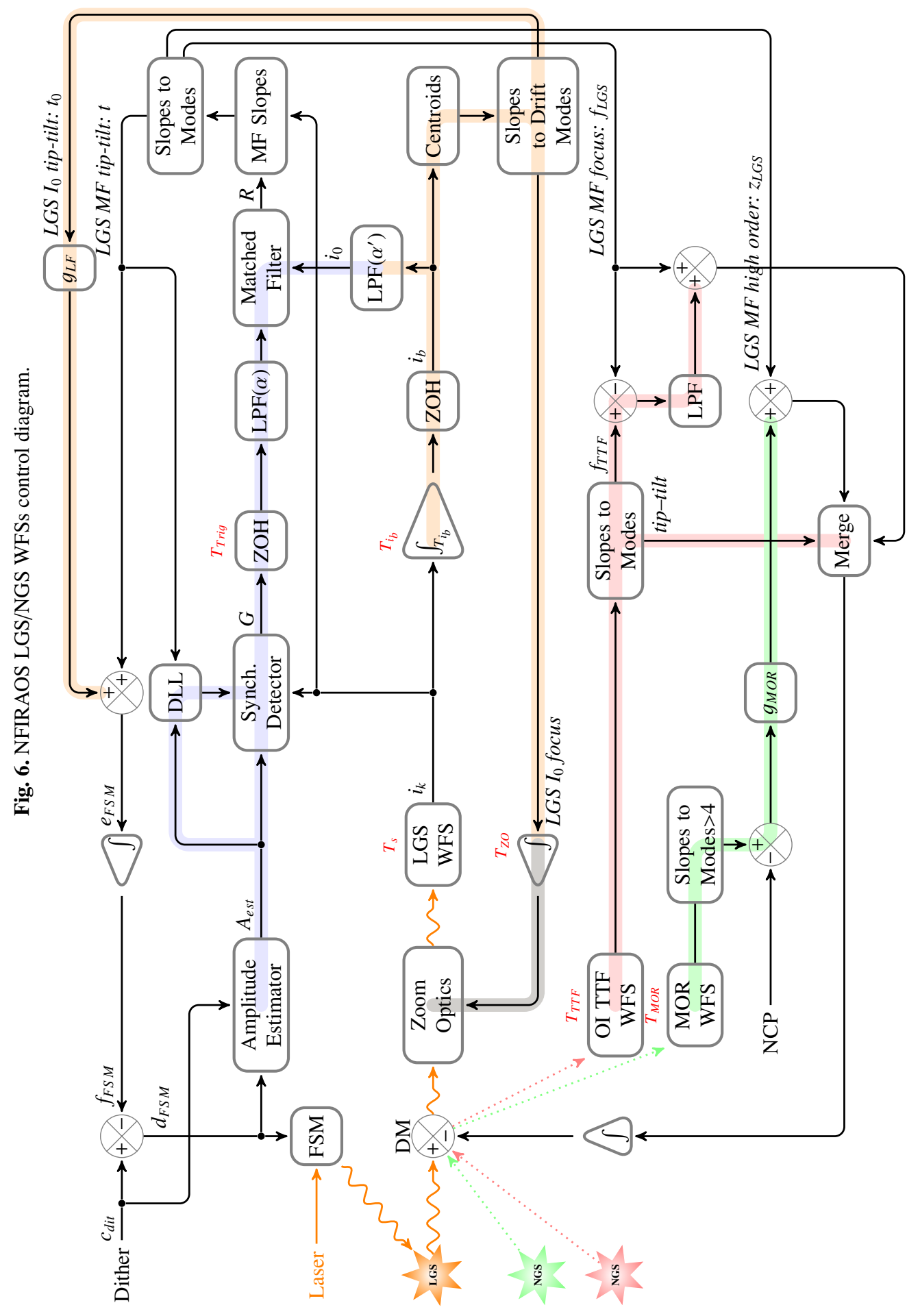


Fig. 7. Theoretical rejection transfer functions.
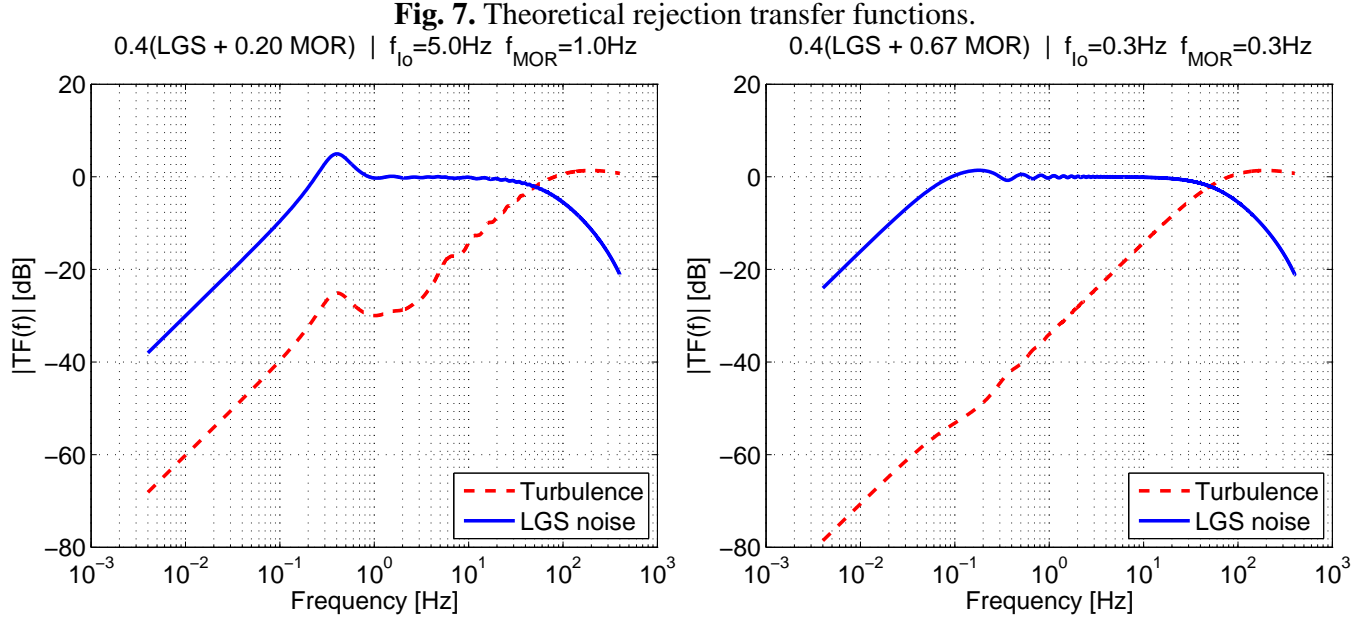

Fig. 8. Bench measured rejection transfer functions.
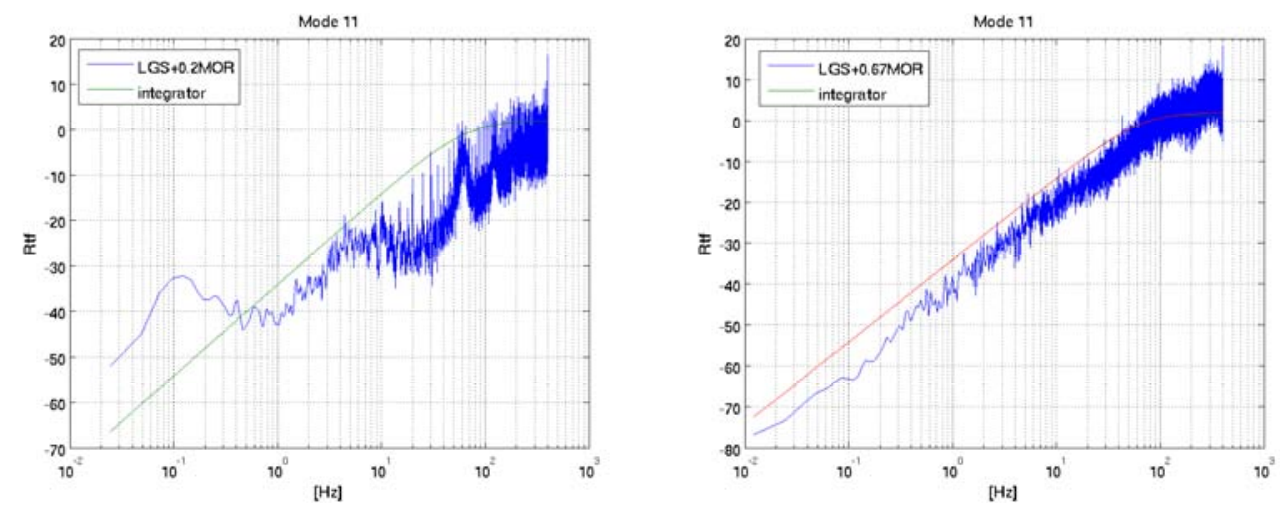

It is worth noting too that the bench can work as an analogical computer for LGS-based tomographic AO. The WFS measurements in different directions can be taken serially and combine afterwards together to reconstruct the turbulence layers. It would be able to take into account the anisoplanaticity of the turbulence and of the Na layer. It could include NGS and LGS WFSs of different orders and with different SNRs.

\section{References}

1. Rodolphe Conan, Olivier Lardière, Glen Herriot, Colin Bradley, and Kate Jackson. Appl. Opt., 48(6):1198-1211, 2009.

2. B. Ellerbroek, S. Adkins, D. Andersen, J. Atwood, C. Boyer, P. Byrnes, R. Conan, L. Gilles, G. Herriot, P. Hickson, E. Hileman, D. Joyce, B. Leckie, M. Liang, T. Pfrommer, J.-C. Sinquin, J.-P. Veran, L. Wang, and P. Welle. volume 7015, Proc. SPIE, July 2008.

3. L. Gilles and B. L. Ellerbroek. Opt. Lett., 33(10):1159-1161, 2008.

4. Luc Gilles and Brent Ellerbroek. Appl. Opt., 45(25):6568-6576, 2006.

5. G. Herriot, P. Hickson, and B. Ellerbroek et al. In Advances in Adaptive Optics II, volume 6272. Proc. SPIE, June 2006.

6. Olivier Lardiere, Rodolphe Conan, Colin Bradley, Kate Jackson, and Glen Herriot. Opt. Express, 16(8):5527-5543, 2008. 